\title{
Trial Manufacture Of Functionally Graded Commutator Made From Copper And Resin
}

\author{
K. Nakano ${ }^{1}$, O. Ishibashi ${ }^{2}$, Y. Sumiya ${ }^{3}$, K. Tateishi ${ }^{4}$, Y. Miyazaki ${ }^{5}$, K. Watanabe $^{6}$, \\ T. Osaki ${ }^{7}$, H. Hasuo ${ }^{8}$ \\ ${ }^{1}$ Kyushu Institute of Technology, JP, nakano@life.kyutech.ac.jp \\ ${ }^{2}$ Otalite Co., Ltd., JP, o-ishibashi@otalite.co.jp \\ ${ }^{3}$ Takada Corporation, JP, sumiya@takada.co.jp \\ ${ }^{4}$ Takada Corporation, JP, tateishi@takada.co.jp \\ ${ }^{5}$ Keisokukensa Co., Ltd., JP, y-miyazaki@keisokukensa.co.jp \\ ${ }^{6}$ Keisokukensa Co., Ltd., JP, k-watanabe@keisokukensa.co.jp \\ ${ }_{8}^{7}$ Fukuoka Industrial Technology Center, JP, oosaki@fitc.pref.fukuoka.jp \\ ${ }^{8}$ Fukuoka Industrial Technology Center, JP, hhasuo@fitc.pref.fukuoka.jp
}

\begin{abstract}
Joining between copper and resin on commutator of electromotor is usually executed by injection molding method and copper is embedded in resin. In this case, clear boundary exists between copper and resin because copper and resin are not combined chemically. This boundary will change to be gaps or cracks after a long time use. In most cases, an abrupt fracture occurs when a force is greater than a certain value applied to this cracks or crack-like defects. In this study, so as to solve this problem, trial manufacture of functionally graded commutator made from copper and resin powder has been executed by spark plasma sintering method. The third element is added into resin powder and resin powder is prepared so that the coefficient of linear thermal expansion may become low. Several mechanical and thermophysical properties have been investigated. The value of shearing stress between copper and copper-resin mixed layer is more than 50Mpa. It shows that the joint strength between copper and resin of this functionally graded commutator without clear boundary is high enough. The more the content of resin increases, the higher the coefficient of thermal expansion becomes. The thermal conductivity in onedimensional and non-steady state at $296 \mathrm{~K}$ indicates that the change is due to the mixing ratio of copper and resin.
\end{abstract}

Keywords: functionally graded commutator, copper, resin, phenol, spark plasma sintering

\section{Introduction}

A commutator of electromotor is usually made by injection molding method. Copper as metallic materials and phenol resin as high polymer materials are joined by integral molding. Its main joining mechanism is an anchor effect. So the amount of copper is used for the anchors in this manufacturing method. In this case, clear boundary exists between copper and resin because they are not combined chemically. This boundary will change to be gaps or cracks after a long time use. Sometimes gaps, which width is a few micrometers, exist on the boundary before use. In most cases, an abrupt fracture occurs when a force is greater than a certain value applied to this cracks or crack-like defects.

In this study, so as to solve this problem, electrolytic copper powder and phenol resin powder have been used as starting materials for the productization of commutator having high reliability. Trial manufacture of functionally graded commutator, which shape is cylindrical and has radial graded composition[1,2], has been carried out considering thermophysical properties by spark plasma sintering method. The third element is added into resin powder and resin powder is prepared so that the coefficient of thermal expansion may become low. Confirmation of axial stress between layers, mechanical property on the boundary between copper and graded layer, and thermophysical properties of graded layers are investigated.

\section{Experimental methods}

\subsection{Used materials}

Starting materials of this functionally graded commutator are copper powder as metallic materials and phenol resin powder as high polymer materials. Electrolytic copper powder is selected for this FGC. Electrolytic copper powder, which shape is like flakes, has good compactibillity but miscibility and flowability of electrolytic copper powder is not so good rather than 
atomized copper powder. So we used fine particle size of electrolytic copper powder, which mean powder particle size is under $50 \mu \mathrm{m}$ and purity is $99.9 \%$.

Phenol resin powder, which number is PG-6551 and produced by Otalite Co., Ltd., Japan, is milled to the almost same size of copper powder by using automatic mortar system before use and glass fiber as the third element is added into phenol resin so as to reduce coefficient of thermal expansion. The aspect ratio of glass fiber is set under 30. Electrolytic copper powder and milled phenol resin powder are mixed by $\mathrm{V}$-form mixing machine according to the mixing ratio for the each layer. Next, compression forming of mixed powder to the green compacts is carried out by compacting machine. After that, spark plasma sintering treatment is executed.

\subsection{Conditions of spark plasma sintering}

(1) Confirmation method of axial stress between layers

So as to check the axial stress between layers during spark plasma sintering, we use pressure sensitive paper, which produced by FUJIFILM Corporation Japan. Two types of the pressure sensitive paper for high pressure and medium pressure are used at a same time. Two sheets of pressure sensitive papers are cut to the ring form and inserted into the boundary of the mixed layer. Coloration and its distribution are checked by using standard chart of momentary stress after pressing.

(2) Conditions of spark plasma sintering

Sintering temperature, heating rate, holding time, and cooling time are set and searched for the optimum sintering condition according to the mixing ratio of copper and phenol resin powder. Two times of spark plasma sintering treatment are carried out for manufacturing one commutator because of the difference of sintering temperature between two materials. The conditions of spark plasma sintering are listed in Table 1 as an example.

Table 1. Conditions of spark plasma sintering

\begin{tabular}{|c|c|c|c|c|c|c|}
\hline No of times & Object & Conditions of spa & lasma sinterin & & & \\
\hline \multirow{3}{*}{1} & \multirow{3}{*}{$\begin{array}{l}100 \% \mathrm{Cu} \\
+ \\
75 \% \mathrm{Cu}\end{array}$} & Temperature (K) & $\mathrm{RT}^{* 1} \rightarrow 773$ & 773 & $773 \rightarrow 473$ & - \\
\hline & & Time (sec.) & 900 & 180 & $\mathrm{FC}^{* 2}$ & - \\
\hline & & Pressure $(\mathrm{MPa})$ & 60 & 60 & 5 & - \\
\hline \multirow{3}{*}{2} & \multirow{3}{*}{ whole } & Temperature $(\mathrm{K})$ & $\mathrm{RT}^{* 1} \rightarrow 393$ & 393 & $393 \rightarrow 573$ & $573 \rightarrow 473$ \\
\hline & & Time (sec.) & 120 & 180 & 300 & $\mathrm{FC}^{* 2}$ \\
\hline & & Pressure (MPa) & 60 & 60 & 60 & 5 \\
\hline
\end{tabular}

(*1)RT:Room temperature, $(* 2) \mathrm{FC}:$ Furnace cooling

\subsection{Evaluation method of mechanical property of the boundary}

So as to evaluate the mechanical strength of the boundary between copper and mixed layer of the functionally graded commutator, which has radial graded composition, we develop the cylindrical shearing test method. Shearing strength is evaluated as strength of the boundary by this testing method. Joint area is measured after spark plasma sintering. Shearing test is executed by using jigs made of austenitic stainless steel. Test specimen form for measuring shear stress is shown in Figure 1. Shearing strength is calculated by the following equation.

$$
\tau=\mathrm{W} / \mathrm{A}
$$

here, $\tau$ : Shearing strength, W:Load and weight of jig, A:Joint area

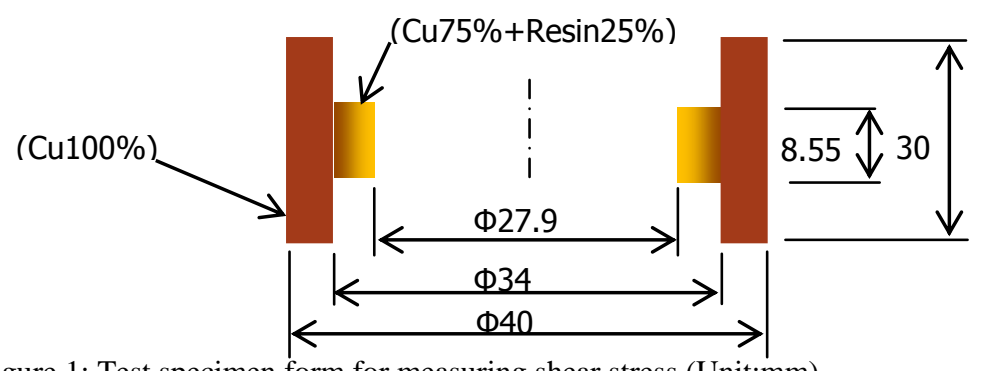

Figure 1: Test specimen form for measuring shear stress (Unit:mm)

\subsection{Evaluation methods of thermophysical properties}

Test specimens of each layer for measuring coefficient of thermal expansion, which size is $5 \mathrm{~mm}$ in width, $5 \mathrm{~mm}$ in length, and $10 \mathrm{~mm}$ in height, are prepared. Thermomechanical analysis method is applied for measuring coefficient of thermal expansion in this study. On the other hand, test specimens of each layer for measuring coefficient of thermal conductivity, which size is $10 \mathrm{~mm}$ in diameter and $3 \mathrm{~mm}$ in thickness, are prepared. The measurements of the thermal conductivity in onedimensional and non-steady state have been executed by laser flash method.

\section{Results and Discussion}

\subsection{External appearance of sintered commutator}


Figure 2 shows external appearance of sintered commutator and schematic figure on sectional view of sintered commutator is shown in Figure 3. The material of the center part, external wall and intermediate graded layer consists of $100 \%$ phenol resin, 100\% copper and mixed material, respectively. Mixing ratio of copper and resin are shown in Figure 3.

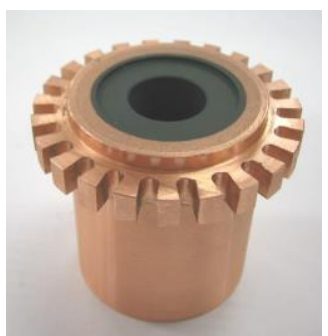

Figure 2: External appearance of sintered commutator

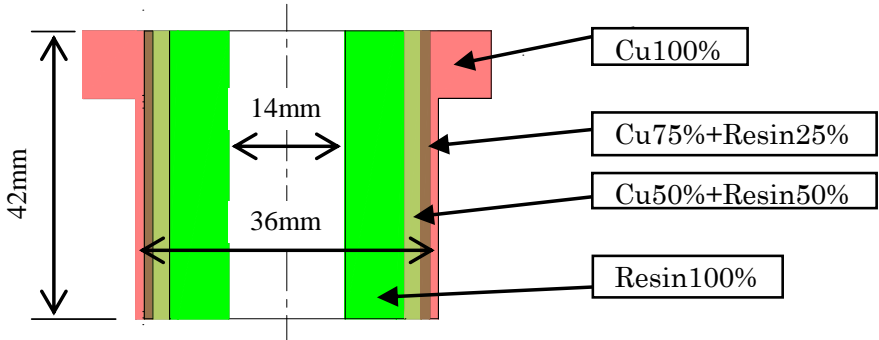

Figure 3: Schematic figure on sectional view of sintered commutator

\subsection{Confirmation result of axial stress between layers}

Figure 4 shows outer appearances of pressure sensitive papers after pressure test. Coloration of the pressure sensitive papers for medium pressure becomes 1.5 in coloration grade, which indicates more than $50 \mathrm{MPa}$ in stress level, by comparing with the standard chart of momentary stress for medium pressure after pressing. On the other hand, coloration of the pressure sensitive papers for high pressure becomes almost 0.3 in coloration grade, which indicates $60 \mathrm{MPa}$ in stress level, by comparing with the standard chart of momentary stress for high pressure after pressing. According to the confirmation results of the axial stress between layers, the stress value indicates almost $60 \mathrm{MPa}$ and it is confirmed that the stress distribution is uniform without bridges.

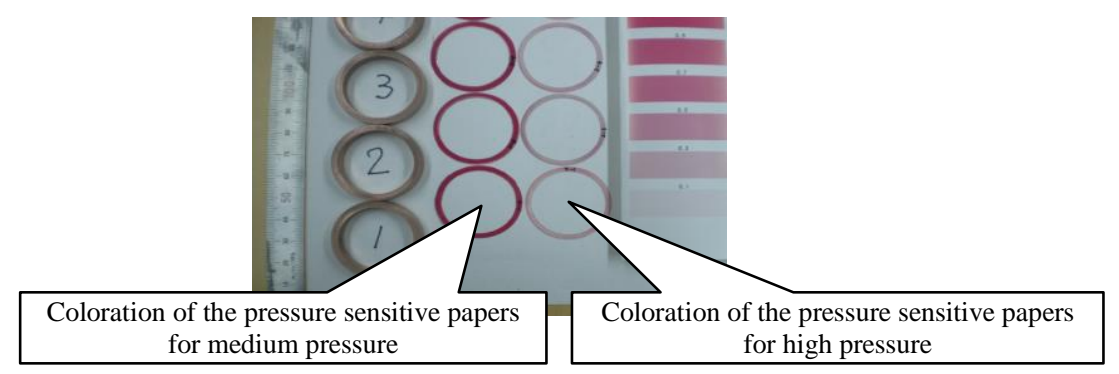

Figure 4: Outer appearances of pressure sensitive papers after pressure test

\subsection{Evaluation result of mechanical property of the boundary}

The results of shearing test are listed in Table 2 and Figure 5 shows SEM image of the copper side fracture surface after shearing test. Shear dimple, which indicates ductile fracture, are observed partially on the copper side fracture surface without machined streaks after shearing test. This result shows that metallic bonding exists just partially on the boundary between copper and graded layer even though the sintering temperature of $773 \mathrm{~K}$ is lower than the melting point of copper at $1356 \mathrm{~K}$. The value of shearing stress indicates more than $50 \mathrm{MPa}$, which is the target value of separation strength. So we confirmed that this joint achieves enough strength.

Table 2. The results of shearing test

\begin{tabular}{ccccc}
\hline Shearing test specimen No. & F11.11.2 & F11.11.4 & F12.15.1 & F12.15.2 \\
\hline Maximum shear stress $\tau(\mathrm{MPa})$ & 53.91 & 67.61 & 69.30 & 69.06 \\
\hline Shearing modulus G $(\mathrm{GPa})$ & 0.509 & 0.634 & 0.600 & 0.740 \\
\hline
\end{tabular}

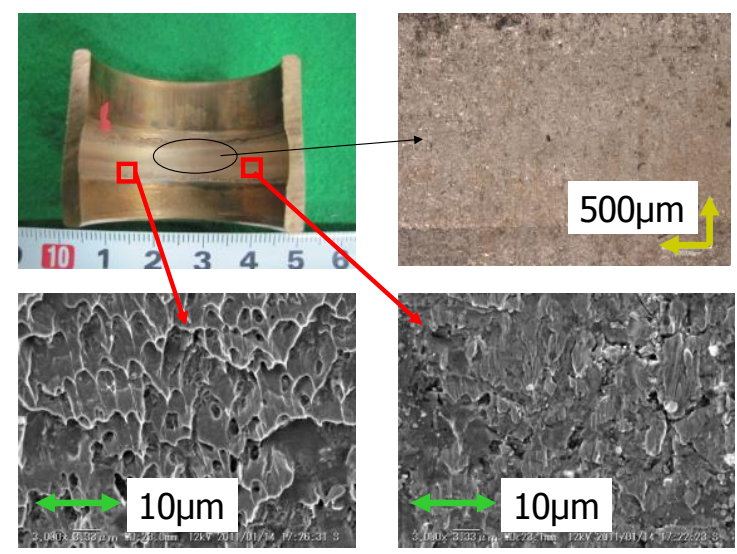

Figure 5: SEM image of the copper side fracture surface after shearing test 


\subsection{Evaluation results of thermophysical properties}

Figure 6 shows the relation between mixing ratio of $\mathrm{Cu} / \mathrm{Phenol}$ resin and coefficient of thermal expansion. The more the content of phenol resin increases, the higher the coefficient of thermal expansion becomes. The changing band of the coefficient of thermal expansion is almost $5 \times 10^{-5} \mathrm{~K}^{-1}$ in case the temperature range is lower than $473 \mathrm{~K}$. And the coefficient of thermal expansion changes so much according to the increase of the content of phenol resin in case the temperature range is over $473 \mathrm{~K}$.

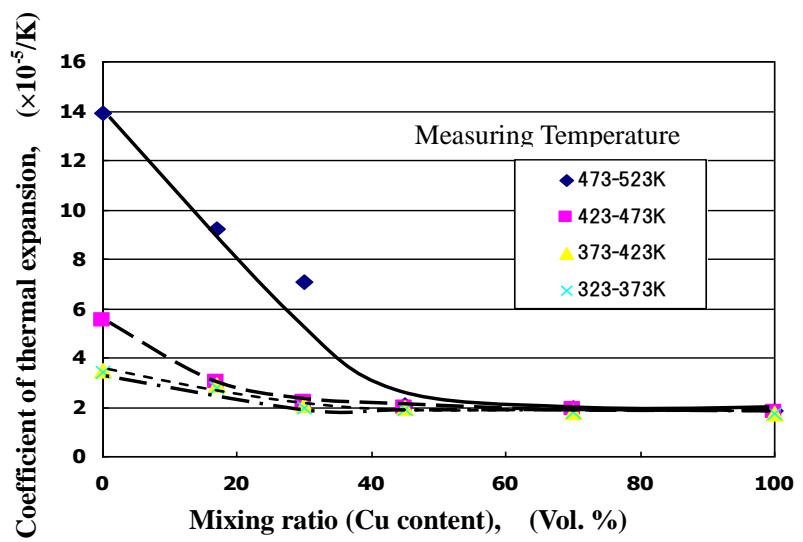

Figure 6: Relation between mixing ratio of $\mathrm{Cu} / \mathrm{Phenol}$ resin and coefficient of thermal expansion

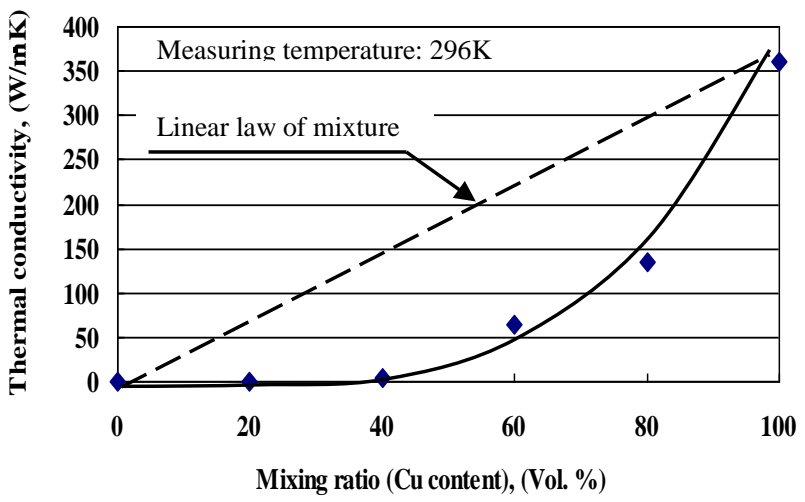

Figure 7: Relation between mixing ratio of $\mathrm{Cu} / \mathrm{Phenol}$ resin and thermal conductivity in one-dimensional and non-steady state at $296 \mathrm{~K}$

Figure 7 shows relation between mixing ratio of $\mathrm{Cu} / \mathrm{Phenol}$ resin and thermal conductivity in one dimensional and nonsteady state at $296 \mathrm{~K}$. The measured value of the thermal conductivity in one-dimensional and non-steady state at $296 \mathrm{~K}$ becomes lower than that based on the linear law of mixture rule. The thermal conductivity in one-dimensional and non-steady state at $296 \mathrm{~K}$ indicates that the monotone increasing change and one to one correspondence according to the increase of copper content. It is confirmed that any value of mean thermal conductivity between copper and phenol resin is able to be designed by changing the thickness of each layer.

\section{Conclusions}

Electrolytic copper powder and phenol resin powder have been used as starting materials for the productization of the commutator having high reliability. Trial manufacture of functionally graded commutator, which shape is cylindrical and has radial graded composition, has been carried out by spark plasma sintering method. Confirmation of the axial stress between layers, mechanical property on the boundary between copper and graded layer, and thermophysical properties of graded layers are investigated. The conclusions obtained in this study are shown as follows:

1) According to the confirmation results of the axial stress between layers, the stress value indicates almost $60 \mathrm{MPa}$ and it is confirmed that the stress distribution is almost uniform without bridges.

2) Shear dimple, which indicates ductile fracture, are observed partially on the copper side fracture surface without machined streaks after shearing test. This result shows that metallic bonding exists on the boundary between copper and graded layer even though it is just partially. The value of shearing stress indicates more than $50 \mathrm{MPa}$, which is the target value of separation strength. It is confirmed that this joint is strong enough.

3) The more the content of phenol resin increases, the higher the coefficient of thermal expansion becomes. The coefficient of thermal expansion changes so much according to the increase of the content of phenol resin in case the temperature range is over $473 \mathrm{~K}$.

4) The thermal conductivity in one-dimensional and non-steady state at $296 \mathrm{~K}$ indicates that the monotone increasing change and one to one correspondence according to the increase of copper content. It is confirmed that any value of mean thermal conductivity between copper and phenol resin is able to be designed by changing the thickness of each layer.

\section{Acknowledgements}

This work was carried out under the FGM project with Fukuoka Industry, Science \& Technology (IST) funding. The authors would like to thank the members of the consortium; Otalite Co., Ltd., Takada Corporation, Keisokukensa Co., Ltd., and Fukuoka Industrial Technology Center.

\section{References}

1. K. Nakano, O. Ishibashi, Y. Sumiya, K. Tateishi, T. Osaki, H. Hasuo, Functionally graded materials and manufacturing methods, JP Patent application No.2010-237669, (2010) (in Japanese).

2. K. Nakano, O. Ishibashi, Y. Sumiya, K. Tateishi, T. Osaki, H. Hasuo, Production methods of functionally graded materials, JP Patent application No.2010239837, (2010) (in Japanese). 